DOI https://doi.org/10.18551/rjoas.2018-11.27

\title{
PLANNING OF HUMAN RESOURCE STRATEGY IN HOSPITAL MANAGEMENT USING THE CONCEPT OF SOCIAL HEALTH INSURANCE SERVICES
}

\author{
Tajus Ibrahim* \\ Graduate Program in Management, Esa Unggul University, Jakarta, Indonesia
}

Tantri Yanuar Rahmat Syah, Lecturer

Faculty of Economic, Esa Unggul University, Jakarta, Indonesia

*E-mail: tajusibr@gmail.com

ORCID: 0000-0002-9859-1640

\begin{abstract}
In Indonesia, it has been stipulated in the 1945 Constitution that the State has a role in protecting its citizens. We must have been familiar to the slogan mentioning that "social security is the responsibility of the state". Health is the most important part of life that characterizes good and prosperous conditions in living a life in which a person's health condition includes healthy body, healthy soul, and socially possible to live productively both socially and economically. Health services must be perceived not only as services to people who are sick, but also insurance for public health as a whole. Health development is a part of national development aiming to improve optimal community health. To fulfill and realize the right of every citizen in obtaining proper health services and fulfill the government's obligations in providing health facilities as mandated by the 1945 Constitution, health is a Public Good, so government's intervention is needed. The potential for hospital development in Indonesia is marked by the current high-level need for health services that can be measured by the degree of public health.
\end{abstract}

\section{KEY WORDS}

Hospital, service management, bundle budgeting, concept of service.

In accordance with the Constitution No.23 of 1999 concerning Health Services, in order to improve health development, the 1982 National Health System was made which is used as a reference in the drafting of State Policy Guidelines on health sector and Constitution No. 23 of 1992 concerning health and also used as a guide on the form and manner of implementation starting from the formulation of policies, guidelines and directions for health development, either by Central and Regional Governments as well as other related parties. Health Services for National Health Insurance, article 1 point 9 (Minister of Health Regulation 71 of 2013), stated that Advanced Referral health services are efforts of individual health services that are specialist or sub-specialist which include advanced outpatient care, advanced level hospitalization and hospitalization in special care rooms.

In 2017, there were 2,755 hospitals in Indonesia which were divided into public hospitals and private hospitals. Public hospitals in Indonesia that are managed by the Ministry of Health were 33 units, Provincial Governments were 134 units, Municipal Governments were 590 units, the Indonesian National Armed Forces/the Indonesian National Police were 170 units, other Ministries were 17 units, SOEs were 87 units, and Private Public Hospitals/Maternity and Children's Hospital were 1724 units (Ministry of Health data, October $23,2017)$. The increasing construction of hospitals by the private sector is also supported by the activeness of the government in encouraging private investment in the hospital business sector. It is basically also related to the limited government funds for the construction of new hospitals. The government has supported the role of the private sector and even foreign investors to play a role in the development of hospitals in Indonesia. However, it was only through a Presidential Decree on the Negative Investment List No. 96 and No.118 of 2000; the government regulated that foreign investors in Indonesian hospital businesses could 
have ownership of up to $49 \%$ of paid up capital. It further encouraged the development of national private hospitals in the form of joint ventures from foreign investors.

Currently, the number of hospitals in Indonesia is arguably in a poor condition since the era of National Health Insurance was held through a mechanism of Social Health Insurance using mandatory NHI programs based on Constitution No.40 of 2004. The aim is all Indonesian people are protected in the insurance system so that they can meet basic public health needs. Initially, hospital services could only be accessed by certain groups that had a lot of money but now it could be accessed by many people. This situation can be seen from the many queues of people who ask to be served, and even take a long time to get services. Behind these lacking services, there is always a good thing or opportunity that we can pursue; building a hospital. In the past, the concept of the hospital was trying to find patients but now it is flooded with patients. However, strong management is required to manage the strategy because when everything is not carefully calculated, it is like a double-edged knife; it will not obtain the benefits in terms of business, instead the big losses.

The growth rate of hospital existence from year to year has increased, especially private hospitals. Previously, in 2015, it was 1,540 units then it increased in 2017 to 1,724 units. It means that business in the health sector is fairly promising and good. In addition, the available data state that Indonesia is still lacking of hospitals and is still far from ideal. Especially at this time, the National Health Insurance (NHI) Program, through Social Security Administrator of Health, has its own large and promising market share for new hospitals to be established. Since the existence of the National Health Insurance through Social Security Administrator of Health, the newly opened hospital will be crowded with patients because all Indonesian people have the right and are guaranteed by the government to get health services in all Health Facilities and Hospitals.

The selection of Bogor District as the location for the business plan of Esummi Hospital describes the private hospital health care services with the advantages of a management system based on patient services of $\mathrm{NHI}$ participant. This business is engaged in the business of healthcare services as a place for quality, effective and efficient prevention and health care providers with core values which include excellence, compassion, respect, delivery, learning and improvement. Health and customer satisfaction are their commitments by always providing excellent plenary services to increase satisfaction and foster patient trust as key customers. It has values (main values) that include: (1) patients are the main customers, (2) good corporate culture, and (3) patient safety.

Based on the above background, it appears that there are still gaps in the huge population of Indonesia which is not balanced with the number of health and hospital service facilities that are not evenly distributed throughout Indonesia. It is a problem for the government about how to build a new hospital or invite private or foreign investors to build hospitals in all regions/regencies in order to fulfill the number of health facilities and fulfill the goals of improving community health status.

Since its implementation in early January 2014 , the NHI program has drawn many pros and cons. There are still many hospital managements that are not ready, are not supported by human resources and information technology systems that are good, reliable, and connected to the Social Security Administrator of Health system. So, there are still many hospitals that carry out administrative processes manually. So far, the obstacle that many $\mathrm{NHI}$ participants complain about is the problem of service, fees, and registration mechanism. Throughout 2014, it was noted that there were more than one hundred thousand complaints regarding the services of $\mathrm{NHI}$ participants. In just three years, the $\mathrm{NHI}$ program certainly still has many limitations and shortcomings, this is tolerable, apart from being a young age, the number of people who have to be dealt with is very large and a very wide area is an obstacle.

Based on the above problem identification, then the problem solving of this business plan includes: (1) building an effective and efficient operational management hospital and a strong team, and providing a clear role for each position, carrying out internal strategies and policies a strong hospital, and having the ability to adapt to the rapid changes in Social Security Administrator of Health rules; (2) providing training and tricks to the coder to get a 
big claim by still prioritizing patient safety; (3) cooperating and integrating with health facilities; (4) implementing a one-day service system and building relationships with Social Security Administrator of Health people; (5) offering and seeking short-term full-timer specialists, and sending the qualified doctors to become specialist doctors; (6) surveying the movement of patients and the business environment around the hospital to be built; (7) making a list of medicines and alternative vendors to overcome the vacancy in the availability of generic drugs (according to the National Formularies) in the E-Catalog (Walker et al, 2015; Gering \& Conner, 2002; Voelker et al, 2001).

The hospital is a unique business category. In addition to its semi-commercial objectives or the elements of scientific development and community service, the hospital currently has an increasing business prospect. The number of the characteristics of a hospital business is a unique business, which does not have the same characteristics with other industry categories (Weingart et al, 2000). It is due to some of the related elements below: (1) The hospital industry is established, of course, not only about profit, but community service. Establishing a hospital is much more difficult than other businesses because it aims at community service. Thus, business plans are made and various activity programs are carried out with the aim of better health services from time to time to the community; (2) Hospital business has very strict rules, either related to licensing and marketing concepts. Similar to the pharmaceutical business or other health services, there are several rules of the game set by the government that aim to protect patients/customers. This requires the hospital to have a unique strategy in its own approach to the community; (3) In the era of global hospital competition, technology is a potential product that has a target market throughout the world. This is an area of excellence that must be met by a global hospital; (4) Excellent service has been embedded in the development of global hospitals. The things needed by the sick are of course a comfortable environment which is formed not only from interior design or other general equipment, but also from the provided services (Ferris et al, 2003; Ayanian\& Weissman, 2002).

\section{HUMAN RESOURCE STRATEGY}

The Law of the Republic of Indonesia Article 35 No. 44 of 2009, concerning Hospitals, mentioned that a hospital is a health service institution for the community with its own characteristics which are influenced by the development of health science, technological advances and socio-economic life of the community which must still be able to improve services that are more quality and affordable by the community to achieve the highest degree of health.

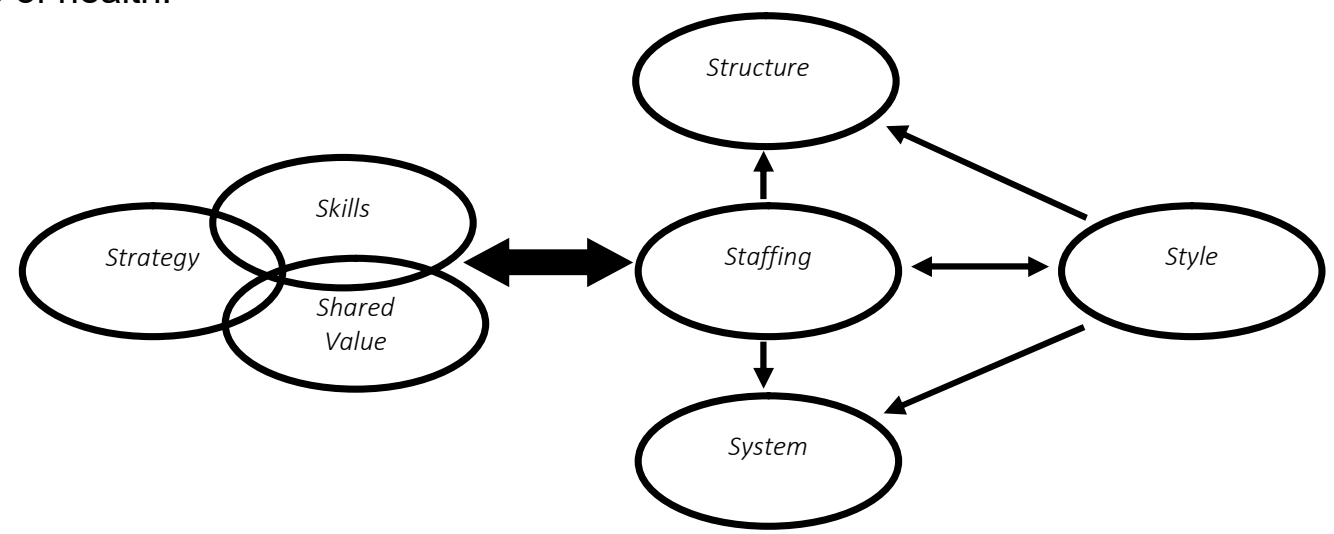

Figure 1 - The 7S McKinsey Model of Esummi Hospital HR

The principles and objectives of the establishment of a hospital must be based on humanity, ethics and professionalism, benefits, justice, equal rights and anti-discrimination, equity, protection, and patient safety and social functions. One of them is the organization of hospitals conducted by health workers who have professional ethics and attitudes and are compliant with hospital ethics (Bamberger et al, 2014). Hospital management is obliged to be 
able to manage human resources, especially health workers, to have competency standards that are appropriate and have professional ethics standards in order to achieve maximum productivity by enhancing their ability and professionalism so that hospital organizations can survive and thrive.

\section{Operational Excellence Strategy for Fast and Excellent Service}

The Operational Excellence Strategy carried out by Esummi Hospital has three basic principles, among others: (1) Construction of fast delivery that emphasizes the administration process in patient services which is accelerated by the booking system. Booking a system in fast delivery is a fast implementation of standard operating procedures that covers the steps when the patient is admitted to the hospital, the patient's service process, and until the patient is discharged from the hospital. Fast and excellent service at the hospital is the service provided to patients so that they are satisfied and want to use the service and will even continue to use the services of the hospital in the future. It is because the services provided by the hospital can really satisfy patients so that patients including their families feel more valuable; (2) The provision of low costs compared to the competitors of the surrounding hospitals, by not reducing the quality of services provided to patients, aims to increase patient visits due to the spread of word of mouth information both patients and their families about the best quality of services received with low costs. For this reason, every patient who enters Esummi Hospital using IHC (Healthy Indonesia Card) will not be charged any fees in any hospital services that are in accordance with NHI-IHC service standards; (3) Providing quality services to patients in which this process brings closer the ways to provide services so that patients may feel comfort and feel being cared for before entering or going out of the hospital. In addition, patients who are about to carry out the patient service process, free from boredom and anxiety. The trick is to provide convenience in the registration process, run a good relationship by always interacting with patients during the ongoing treatment or providing health information about health services, receiving patient criticism and suggestions that can be directly delivered via telephone, SMS, BBM (Blackberry Messenger), e-mail, and WhatsApp including the process of scheduling patient services or actions (Bowen \& Ford, 2004; Mok et al, 2013).

There are several workforce activities in Esummi Hospital that must be applied to the patient care in a hospital, including: (1) Creating reliable human resources is the best step to improve and create professional service excellence in a hospital. Reliable human resources can be achieved by education and training on a regular basis and in accordance with the fields of each officer; (2) Making hospital workers responsive or taking immediate action before being asked by the patient, as well as being skilled in accordance with their competence and being able to respect their patients so that the patient will use the services of the hospital again. The workers in this case include first-line officers who are dealing with patients until the patient is handled by the DPC (Doctor of Patients in Charge). To achieve this goal, every worker in the hospital from the executive level to the director board level must take part in Service Excellence training both internally and externally. Therefore, all activities taking place in the hospital will become hospital operational activities; (3) Making the workers in the hospital skillful and be able to respect their patients so that the patient will always return to use the services at the hospital; (4) Creating competence as one of the hospital's important operational excellence activities. By applying these competencies, all workers in the hospital can master their duties properly and professionally.

\section{Casemix Skill and Lean Competency}

In an effort to maintain the quality of medical care and nursing care, monitoring and quality control of the profession on an ongoing basis needs to be carried out. For this reason, efforts to monitor and control professional quality are carried out through: (1) Quality monitoring, for example by using Morning Report, difficult case discussions, rounds of rooms, death cases, medical audits and Journal Reading; (2) Follow up on quality findings such as short training, continuing education activities, and additional authority education. There is a monitoring of the quality of service of medical staff in hospitals, it is expected that unexpected events in terms of patient safety can be prevented. Hospital management together with the Medical Committee establishes matters that are evaluated in the service of 
medical staff through coordination work meetings. Based on the results of the coordination meeting of the Medical Committee, there were two main things evaluated at the hospital medical staff that include: on-going professional practice evaluation and focused professional performance evaluation. Included in the on-going professional practice evaluation are: (1) Ethics and communication in the coordination of work among doctors, management and patients; (2) The discipline of the doctor during the patient's care in outpatient section/polyclinic; (3) The discipline of the doctor when patient service is outpatient section (time of visit and number of visits); (4) Compliance of doctors in the implementation of the Clinical Pathway; (5) Completeness of filling in medical record files (filling in medical resumes, consultation sheets, etc.); (6) Pattern of blood use and medicines/pharmacy; (7) Pattern of treatment duration; and (8) Utilization of consultation. Meanwhile, those included in focused professional performance evaluation are: (1) Review of the existence of Unexpected Events or Sentinel conducted by the case study in the form of discussion of difficult cases, discussion of death cases and medical audits; (2) Patient complaints against the doctors.

Patient service management from the concept of patient-focused services consists of four elements, among others: (1) It requires health care providers to listen and respect the views and choices of patients and families. In addition, knowledge and values, beliefs, cultural backgrounds of patients and families are included in the planning of health services and services; (2) It requires health care providers to communicate and share information thoroughly with patients and families, in which patients and families also receive timely, complete and accurate information; (3) Patients and families are encouraged and supported to participate in care and decision making and their choices; (4) Patients and families are partners of health care providers who require health care providers to collaborate with patients and families in the development, implementation and evaluation of policies and programs.

Currently, patient-centered care applies a more modern and innovative approach to health care. It is no longer doctor-centered, but a team leader of an interdisciplinary team, with adequate competence, in which each patient caregiver is equally important in health care for patients. A case manager is a person outside the patient care provider, whose job is to coordinate each patient care provider, so that patients get integrated services according to their needs. The integration and coordination of patient care activities is the goal to produce an efficient care process. Examples of team care are implemented in the form of: patient safety rounds between sections in the hospital, a combination of care planning forms, integrated patient medical record files, and a case manager (Banham \& Connelly, 2002). Case managers must have a professional working relationship with doctors and clinical staffs. They must also be familiar with billing services, financial assistance services, assistance/support from the community and spiritual services. It is important for case managers to have relationships with patients and families. Case managers need to maintain mutual trust to show the patients that they are involved in patient benefits and interests. In the implementation of patient service management, a case manager can handle 25-70 patients, depending on the condition of complexity, clinical service system, and hospital work culture. Case managers conduct screening to patients who need patient service management based on high risk patients, high costs, high complaints potential, and cases with chronic, complex or complicated diseases. Case managers draw up the patient service management plan and collaborate with the Doctor of Patients in Charge and other clinical team members which reflects the appropriateness and cost-effectiveness of medical and clinical treatment and the patient's decision-making needs. There is documentation evidence of case manager activities which are included in medical records such as recording in information education forms.

Case Managers must immediately identify short-term, long-term and ongoing needs so that patient service management strategies and objectives can be developed to meet those needs. It is also necessary to prepare facilities, overcome problems and conflicts and pay attention to the expectations of payers, frequency of communication re-evaluation of patient development, revision of short and/or long-term goals. 


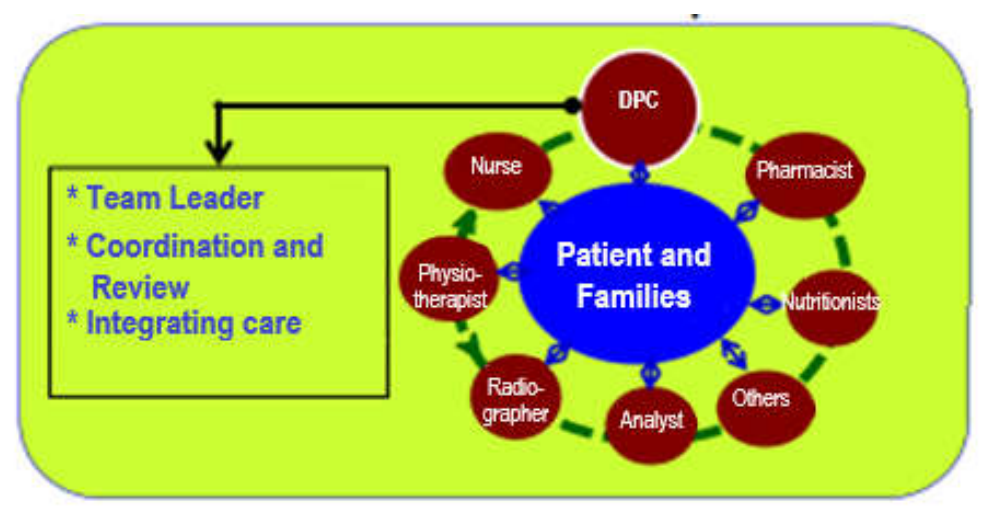

Figure 2 - Coordination Diagram of Service Continuity Integration in Interdisciplinary Care Team

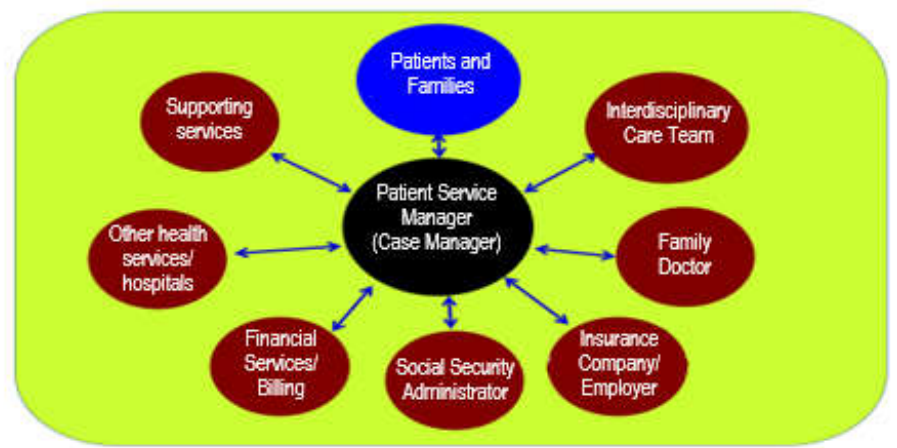

Figure 3 - Coordination Diagram of Continuity in Patient Service Management

Shared Value: Continuous Improvement \& Knowledge Management. Esummi Hospital Operations carry out Continuous Improvement, which is an ongoing effort to develop and improve products and patient service processes in a hospital. The business aims to find and get the best form of the resulting improvement. Creating the best solution of the problem, which often arises during the patient service process, the results of which will continue to survive without any improvement in service and or develop better with the improvement of the service system both in technology and competency of the patient service workforce; depends on the assessment of patient satisfaction level as long as they get health care at the hospital.

There are eight easy steps to conduct Continuous Improvement activities, including: (1) The number of problems faced by an organization or company can be mapped through the problem matrix by providing value based on the urgency of the problem using the Pareto diagram approach to determine the frequency of problems and determine the dominant parameters; (2) Collecting the data related to problems that will be resolved with clear and valid data; (3) In searching for the root of the problem, the Fishbone Diagram method or also known as Cause-Effect Diagram or the 5-Why approach can help in identifying various potential causes of a problem. It is carried out by gathering people who are relevant and have a spirit of improvement and then asking them five things related to why a problem can occur; (4) After understanding the problem, it is formulating an alternative repair in the form of a matrix so that it is easy to see the best solution by setting two or three alternative improvements to be carried out; (5) Applying a solution in a location or place according to the data available, then taking the measurements in which the results are based on the initial data period, for example a week, a month or three months; (6) Performing a conformity analysis with the target to be achieved. If it is not appropriate, then it is going back to step three (look for the root of the problem). If it is appropriate then it can proceed to step seven; (7) Making standardization in the form of SOP (Standard Operating Procedures) if the targeted results are achieved; (8) Finding a new problem (Continuous improvement) by doing more mapping of new problems to continue to make improvements. 
One of the efforts to deal with all the problems of patient care in this hospital is to change the mindset of all individual officers who consider that efforts to improve the hospital are as a series of 'seasonal' activities (because of the distance between limited time and resource). When the hospital has decided to implement a culture of continuous improvement in all work units, the hospital must realize that the success depends on a series of hard work efforts from all the involved resources which are carried out continuously. Hospital industry, whose work unit is closely related to health services, has two main categories in its business activities. The first category relates to the patient service process, which refers to all work, either proactive and reactive, and focuses on short-term outcomes. The second category deals with increasing operational activities to be more effective and efficient which refers to jobs that make operating activities more efficient, and focus on long-term results. Both activities are a challenge as well as a priority agenda for hospitals in implementing continuous improvement efforts. What is needed by the hospital is a systematic approach that is carried out continuously so that an activity that will become a culture in the hospital service system can be embedded. The effort to implement continuous improvement culture at the hospital is by introducing to all hospital staff that the improvement effort is not about a method or a set of tools that are able to solve the existing problems in a short time (Winkelman, \& Choo 2003).

Esummi Hospital knowledge management activities are aimed at achieving certain outcomes such as shared knowledge, improved performance, competitive advantage, or higher levels of innovation. The concept of knowledge management includes HR management and information technology (IT) whose purpose is to reach a good hospital organization so that it can win business competition. The development of information technology plays an important role in the concept of knowledge management which cannot be separated from the management of patient services in the hospital. This condition requires new ways of addressing all the problems of hospital activities that occur in order to survive. The importance of HR quality is one of the responses in addressing these changes, and this of course requires efforts to improve and develop HR. In connection with this matter, the role of science is becoming more prominent because only with knowledge all the changes can be addressed appropriately. It means that education plays an important role in preparing quality and competitive human resources.

The implementation of knowledge management will have a positive influence on the company's business processes directly and indirectly, some of the benefits of knowledge management for companies include: (1) With a well-structured knowledge source, the company will be easier to use that knowledge for other contexts, so that the hospital can save time and money; (2) The source of knowledge will provide convenience to each hospital staff to utilize it, so that the process of utilizing knowledge in the hospital environment will increase; in the end of process, creativity and innovation will be encouraged more broadly and each hospital staff can improve their competence; (3) The hospital will be able to easily adapt to changes in the business environment; (4) Existing knowledge can be reused for the process or service to be developed, so that productivity of the hospital will increase.

Organizational Structure: Cross-Functional. Organizations can be seen from two different angles, including: (1) The structure in which the business environment requires customer-focused structures and cross-functional collaboration; (2) Capabilities, in which the business environment requires organizations to have the capability to learn, the capacity to change and have high accountability. Presidential Regulation of the Republic of Indonesia No. 77 of 2015, concerning effective, efficient and accountable hospital organization guidelines are implemented in order to achieve the vision and mission of the hospital in accordance with good corporate governance and good clinical governance. The structure of the hospital is considered to have an easy range of control because the hospital director can directly provide supervision to the direct staff, as well as the medical, nursing and medical support departments. Therefore, the level consists only of hospital directors, medical service divisions, nursing service divisions, general and financial divisions, and committees. It is intended to be able to cut operating costs which are certainly in accordance with government regulations and are able to work effectively. Esummi Hospital saw three aspects that exist in 
a structure, including: (1) Puller (puller) is the part that provides input, namely the medical and nursing services division; (2) Core is the operational part of the hospital; the executive nurse, general practitioner and specialist; (3) Support is part of Puller and Core support so that both of these things can carry out their functions optimally. Support is part of the support in carrying out hospital operations; general and financial parts. These three aspects form a cross functional team which is a group of personnel who come from various functions or disciplines within an organization that has the same goal.

Esummi Hospital carries out the principle of Cross Functional Organization, in which the organization is oriented to a system that prioritizes patient service needs as a customer and the application of HR in forming Cross Functional Team in which each team has the responsibility to realize the system goals by making continuous improvements to the system. The Cross Functional Paradigm places the organization as a series of systems that are used to serve customer needs, as well as a collection of shared competencies and resources provided to meet customer needs. To deal with the ever-changing business environment conditions, Esummi Hospital must have a learning organization where all personnel have the ability to adapt to the trend of change and be able to adjust competencies to meet the demands of changes which will potentially be the determinants of organizational survival. Cross Functional Organization has advantages in terms of speed and complexity which focus on satisfying customer needs, creativity, learning and single point of contact.

Staffing: Job Description. In carrying out health service operations, Esummi Hospital which employs doctors and nurses must also be able to carry out work with existing job descriptions in which the assessment benchmark is in accordance with what the company's expectation with the provision of work assessments made twice a year. Basically, the job description is a formal document of the organization which contains a summary of important information about a position to facilitate differentiating positions from one another in Esummi Hospital. This job description is arranged in a structured format so that information is easily understood by all parties involved in this company. Job descriptions at Esummi Hospital must be clearly defined for each position, so that the official knows the duties and responsibilities he/she must do. Job description will provide assertiveness and standard tasks that must be achieved by an official who holds the position. Meanwhile, competence is a standard that must be fulfilled from a position. It is also supported by supporting/expert staff as advisory boards, who are also responsible for services and can provide operational advice and technical assistance at the hospital.

In order to achieve its objectives, in realizing its existence, an organization requires effective HR planning. Human resource planning is a management process in determining the movement of organizational human resources from a desired position in the future, while human resources are a set of processes and activities carried out jointly by HR managers and line managers to solve organizational problems that related to humans. The unification and coordination of human resources in hospitals requires human resources with the ability and mastery to cover aspects of management, legal aspects, and organizational culture. The comprehensiveness of this aspect of HR needs to be studied and developed in conjunction with the physical development of the hospital based on a framework of thought regarding the medical / epidemiological feasibility of the Esummi Hospital project. Therefore, HR needs can be expressed in the following sections: (1) HR needs for public services in hospitals; (2) HR needs for superior hospital services are in accordance to the planning. Even so, new hospital HR planning for both medical and non-medical human resources including recruitmentretention-release strategies of each HR need is made in more detail in the preparation of the Master Plan.

The addition of the availability of hospital operational workforce from the implementation level to the director level is discussed at the monthly HRD meetings scheduled for the third week. The HRD meeting is attended by all managers and directors only, where previously there was a workload analysis record made by the manager related to the addition of staffs at Esummi Hospital according to the needs of units/ sections. In the workforce recruitment, hospital prioritizes the community around the hospital and prioritize the families of the workers who have worked firstly in the hospital. However, it is still through 
the selection of recruitment teams from hospital HRDs by prioritizing competencies that are in line with job formation.

Systems: Lean Management \& Customer Relationship Management. Lean management is required in the work process in the entire value chain of the organization so that various forms of potential waste can be prevented. The hospital, which focuses on the operation of health services specifically for $\mathrm{NHI}-\mathrm{IHC}$ participants, has several ways so that the process can provide maximum service, including the application of Lean Management function in the Blue Print Service, in which the goal is to simplify process and dispose of waste in the process in order to be able to provide better services from other existing places.

There are four operational "times" that must be considered and carried out by the hospital workers in the work process so that patients feel the comfort and satisfaction of medical services, among others: (1) On time which means that the DPC service at the clinic is always on time or comes before the practice hours begin; (2) Response time which means that in handling medical emergencies or patient complaints for medical or general problems, it is immediately/quickly responded to and followed up according to the problems of the patients or their family; (3) Waiting time means that it does not make the patients or his family wait in a long time to get medical services, medical information and/or handling complaints from the patients regarding medical/general services at the hospital; (4) Time motion means to shorten the service time experienced by the patients from the beginning process in the hospital until being examined and following up on the medical treatment until the patient returning home feeling satisfied with the services provided by the hospital. In supporting these processes and systems, Esummi Hospital is also assisted by a marketing system using CRM (Customer Relationship Management) in which this system is a marketing tool that builds close relationships between companies, i.e. hospitals and patients. In this system, it is expected that various service matters at Esummi Hospital related to planning, implementation, control and evaluation, budgets, and awards can be run optimally by the hospital.

Style: Transformation. The leadership style applied at Esummi Hospital is situational leadership that focuses on two basic concepts, namely the combination of leadership style and the development stage of the individual or group maturity level. According to Harsey and Blanchard, there are four leadership styles (S1 to S4) that are adjusted to the stage of developing worker ability (D1 to D4). It is expected that the hospital is able to run this leadership style model, starting when the company is established, in accordance with the existing timeframe, to be able to become a hospital that is able to optimal service, and be able to develop its employees as the core of the company's success.

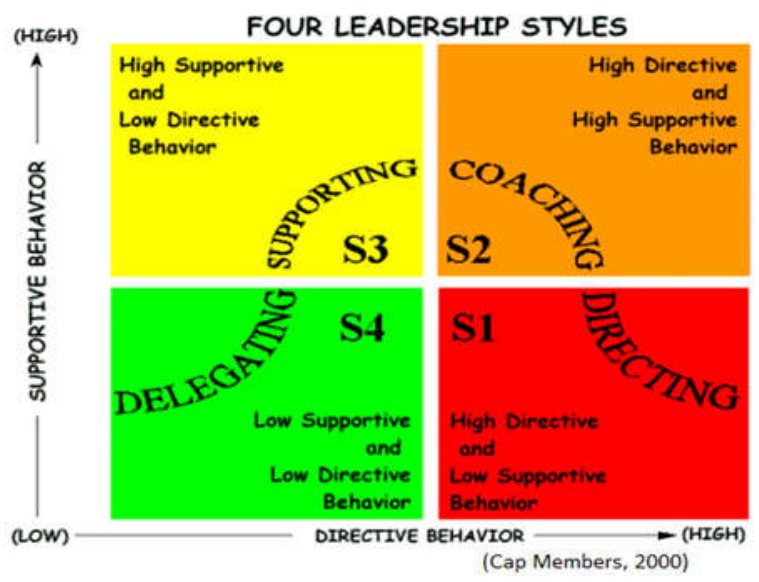

Figure 4 - Leadership Style

Leadership Situation S1 (Telling/Directing). In this situation, all hospital workers are still in the initial process, who do not know the pattern of good and fast work, and the tasks run very long because they are still hesitant to try something new. So, the leader must carry out 
a role to direct. At this stage, attention is still intended to develop employee competence that is practically not well developed. Basically, in this situation, employees only do what is ordered by the leader.

Leadership Situation S2 (Selling/Coaching). This situation occurs when all hospital workers still lack competence, but they have the desire to work and want to try new things. In this situation, the hospital director has to give advice more on the implementation of various jobs than to instruct the employees to do the work in detail. Therefore, the leader must try to "sell" various ideas about how to carry out more effective and efficient work that so that the employee motivation can be further enhanced in which they can complete the task properly and correctly.

Leadership Situation S3 (Participating/Supporting). In this situation, all hospital staff have high competence, but they have a feeling of insecurity to do the job. In this situation, the leader must show what must be carried out by the employees and ask the employees to work together to carry out the work of their duties because they have the ability to do the work. In this situation, the hospital director must also motivate/encourage the hospital employees with the aim of increasing their confidence so that they are able to carry out their duties.

Leadership Situation S4 (Delegating/Observing). In this situation, all the officers in Esummi Hospital had the competence and also high commitment to complete the task so that the leader could delegate the work to the employees. Leaders in this situation have a focus on work and a low working relationship with the employees. Employees in this situation need little support from leaders because they can work independently. It is expected that this situational model leadership pattern can be implemented.

The rapid development of medical technology, the shift in disease patterns, the fluctuating changes in government policies in $\mathrm{NHI}-\mathrm{IHC}$, and all of these dynamic conditions have caused the hospital business environment to become increasingly complex and rapidly changing. Regarding to these conditions, every hospital is required to immediately change or carry out transformation and adapt to changes in the hospital business environment that was originally a competitor and then became a partnership. This transformation process is carried out through the transformation of patient service governance as well as the transformation of human resources. Quality control and cost control are carried out in the transformation of patient service governance. Meanwhile, the transformation of the HR function is intended to produce HR who have the capability to participate in the organizational change process.

\section{CONCLUSION}

Esummi Hospital Operations carry out Continuous Improvement and Knowledge Management which aims to find and get the best form and solution of problems that often arise during the patient service process, which results will continue to survive without any improvement in service and or develop better with the improvement of the system technology service and worker competency to the patient service. It depends on the assessment on the level of patient satisfaction when they get health care at the hospital.

Esummi Hospital carries out the principle of Cross Functional Organization, in which the organization is oriented to a system that prioritizes patient service needs as the customer and the implementation of HR that forms a Cross Functional Team where each team has the responsibility to realize the system goals by making continuous improvements to the system. The Cross Functional Paradigm places the organization as a series of systems that is used to serve customer needs as a collection of shared competencies and resources that is provided to meet customer needs. To deal with the ever-changing business environment conditions, Esummi Hospital must have a learning organization in which all personnel have the ability to adapt to the trend of change and are able to adjust competencies to meet the demands for changes that occur or which will potentially occur; it will be the determinants of survival of the organization. Cross Functional Organization has advantages in terms of speed, complexity, focusing on satisfying customer needs, creativity, learning and single point of contact. 


\section{SUGGESTIONS}

Human resource management in a hospital should be carried out by building the character of hospital workers to have the senses of organization and competencies that are always upgraded and prioritize the safety of patients in the health service.

\section{REFERENCES}

1. Walker, J., Pan, E., Johnston, D., Adler-Milstein, J., Bates, D. W., \& Middleton, B. (2005). The Value of Health Care Information Exchange And Interoperability: There is a business case to be made for spending money on a fully standardized nationwide system. Health affairs, 24(Suppl1), W5-10.

2. Gering, J., \& Conner, J. (2002). A strategic approach to employee retention. Healthcare Financial Management, 56(11), 40-44.

3. Voelker, K. E., Rakich, J. S., \& French, G. R. (2001). The balanced scorecard in healthcare organizations: a performance measurement and strategic planning methodology. Hospital topics, 79(3), 13-24.

4. Weingart, S. N., lezzoni, L. I., Davis, R. B., Palmer, R. H., Cahalane, M., Hamel, M. B., ... \& Banks, N. J. (2000). Use of administrative data to find substandard care: validation of the complications screening program. Medical care, 796-806.

5. Ferris, T. G., Marino, B. L., Homer, C. J., \& Perrin, J. M. (2003). Are hospital characteristics associated with parental views of pediatric inpatient care quality?. Pediatrics, 111(2), 308-314.

6. Ayanian, J. Z., \& Weissman, J. S. (2002). Teaching hospitals and quality of care: a review of the literature. The Milbank Quarterly, 80(3), 569-593.

7. Bamberger, P. A., Meshoulam, I., \& Biron, M. (2014). Human resource strategy: Formulation, implementation, and impact. Routledge.

8. Bowen, J., \& Ford, R. C. (2004). What experts say about managing hospitality service delivery systems. International journal of contemporary hospitality management, 16(7), 394-401.

9. Mok, C., Sparks, B., \& Kadampully, J. (2013). Service quality management in hospitality, tourism, and leisure. Routledge.

10. Banham, L., \& Connelly, J. (2002). Skill mix, doctors and nurses: substitution or diversification? Journal of Management in Medicine, 16(4), 259-270.

11. Winkelman, W. J., \& Choo, C. W. (2003). Provider-sponsored virtual communities for chronic patients: improving health outcomes through organizational patient-centred knowledge management. Health expectations, 6(4), 352-358. 